livraisons

d'Histoire

de l'Architecture

\section{Livraisons de l'histoire de l'architecture}

41 | 2021

L'architecte en son agence

\title{
Nicodeme Tessin l'Aîné et Jean de la Vallée, les premiers architectes en Suède et leurs agences
}

Nicodemus Tessin the elder and Jean de la Vallée: the first architects in Sweden and their firms

Nicodemus Tessin der Ältere und Jean de la Vallée, die ersten Architekten mit Büros in Schweden

Linnéa Rollenhagen-Tilly

\section{OpenEdition}

Journals

Édition électronique

URL : https://journals.openedition.org//ha/3030

DOI : $10.4000 /$ /ha.3030

ISSN : 1960-5994

Éditeur

Association Livraisons d'histoire de l'architecture - LHA

Référence électronique

Linnéa Rollenhagen-Tilly, « Nicodeme Tessin l'Aîné et Jean de la Vallée, les premiers architectes en Suède et leurs agences ", Livraisons de l'histoire de l'architecture [En ligne], 41 | 2021, mis en ligne le 15 juin 2021, consulté le 20 juin 2021. URL : http://journals.openedition.org//ha/3030 ; DOI : https:// doi.org/10.4000/lha.3030

Ce document a été généré automatiquement le 20 juin 2021

Tous droits réservés à l'Association LHA 


\section{Nicodeme Tessin l'Aîné et Jean de la Vallée, les premiers architectes en Suède et leurs agences}

Nicodemus Tessin the elder and Jean de la Vallée: the first architects in Sweden and their firms

Nicodemus Tessin der Ältere und Jean de la Vallée, die ersten Architekten mit Büros in Schweden

Linnéa Rollenhagen-Tilly

\section{NOTE DE L'AUTEUR}

Cet article relève d'une étude en cours financée par Berit Wallenbergs stiftelse et Åke Wibergs stiftelse.

1 Après la guerre de Trente ans (1618-1648), la Suède compte parmi les grandes puissances en Europe. En quelques décennies, Stockholm se métamorphose en une ville européenne et des chantiers ambitieux démarrent dans tout le royaume. Les architectes Nicodème Tessin l'Aîné (1615-1681) et Jean de la Vallée (1624-1696) sont mentionnés dans de nombreux travaux portant sur cette période de construction intense. Les lignes qui suivent proposent une synthèse analytique des connaissances que nous pouvons en extraire, concernant les manières dont ils pratiquaient le métier d'architecte. Depuis le début du $\mathrm{XX}^{\mathrm{e}}$ siècle, des générations d'historiens de l'art ont exploré des archives abondantes, impossibles à appréhender par un chercheur seul. Les ouvrages de référence d'Oswald Sirén et de Ragnar Josephson - deux monographies, sur Tessin l'Aîné et son fils, ainsi qu'une synthèse sur la construction de Stockholm avant $1800^{1}$ - ont été prolongés par maints chercheurs, notamment dans le cadre d'inventaires d'églises et de châteaux suédois, ainsi que dans des monographies plus récentes sur Jean de la Vallée et Nicodème Tessin l'Aîné ${ }^{2}$. Ces travaux s'appuient 
notamment sur l'ouvrage Suecia Antiqua et Hodierna et ses dessins préparatoires, conservés à la bibliothèque royale de Stockholm, qui constituent une source inestimable. Entrepris et supervisé par Erik Dahlbergh au début des années 1660, ce recueil forme un panorama architectural et urbain illustré de la Suède du XVII siècle et présente l'essentiel de l'œuvre de nos deux $\operatorname{architectes}^{3}$. S'il est impossible de reconstituer les modalités précises de leur production architecturale, classer et croiser des informations publiées permet de mettre en évidence l'introduction de nouvelles pratiques du métier d'architecte, et laisse supposer que c'est par leur notoriété que Nicodème Tessin l'Aîné et Jean de la Vallée contribuèrent à la naissance d'un nouveau statut et d'une nouvelle organisation de leur profession. Après un bref historique de leurs origines et formations, cet article montrera en quoi leurs méthodes de travail permettent de les présenter comme les premiers architectes et donnera un aperçu de ce que l'on peut entendre comme les premières agences d'architecture en Suède.

\section{Origines et formations de deux architectes phares}

2 Nicodème Tessin l'Aîné est né en 1615 dans une famille de notables installée à Stralsund en Poméranie, où il semble avoir reçu un enseignement des fondamentaux de lecture, d'écriture et de mathématiques dans les années $1620^{4}$. L'alliance de la Poméranie avec la Suède en 1628 permettait la remise en état des fortifications de Stralsund. Orphelin de père dès 1632, il est plausible que Tessin eut son premier apprentissage sur ces chantiers suédois; il fut en tout cas engagé par la Fortification suédoise en tant qu'ingénieur et copio conducteur (conducteur de chantier) en $1636^{5}$. Il est également possible que Tessin ait suivi en Suède, dès cette même année 1636, son supérieur, Olof Hanson Örnhufvud (1600-1644), quartier-maître général des châteaux et fortifications suédoises. Néanmoins nous ne disposons d'aucune information sur l'activité de Tessin avant mars 1639, où un passeport établi à son nom fait état de " frais de voyages" probablement effectués pour le grand chancelier Axel Oxenstierna (1583-1654) ${ }^{6}$.

3 Quant à Jean de la Vallée, il est né à Paris vers 1623-1624, dans une famille bien établie dans le milieu de la construction. Il quitte la France en 1633 et séjourne d'abord dans les Provinces Unies où son père, le maître maçon Simon de la Vallée (1596-1642), avait reçu le titre officiel d'architecte du prince d'Orange. Recruté par le maréchal suédois Åke Tott (1598-1640), le père émigre avec ses deux fils en Suède au printemps 1637. Avant d'être nommé architecteur de sa Majesté en 1639, Simon de la Vallée obtint des commandes des familles notables Oxenstierna et de la Gardie. À cette époque, il y a une distinction en suédois entre architecteur et architecte, l'activité du premier implique une activité physique et manuelle (présence sur le chantier), alors que le second titre suppose une approche plus intellectuelle. Le 21 avril 1640, Tessin fut missionné par Axel Oxenstierna pour organiser et superviser les travaux dans ses châteaux, devenant ainsi le chef de chantier en vue de l'exécution de dessins de Simon de la Vallée ${ }^{8}$. Il est vraisemblable que Jean de la Vallée commença, ou poursuivit, un apprentissage auprès de son père et/ou de Tessin.

4 Simon de la Vallée décéda brusquement, à la suite d'une agression, en décembre 1642. Le mois suivant, l'agresseur, le colonel Erik Oxenstierna, promit de contribuer à ce que "Jean Lavalet puisse disposer de quelque bénéfice de la Couronne afin de pouvoir continuer son art(isanat) dans les meilleures conditions, et à l'avenir se rendre utile au 
service de la Couronne ${ }^{9}$ ». Ainsi Jean de la Vallée obtint une subvention de la royauté pour son éducation et logea chez le gouverneur Klas Fleming (1592-1644). Cette période correspond à ses premiers "travaux " documentés et rémunérés, notamment des relevés de bâtiments pour Axel Oxenstierna et la conception des plans du corps principal d'un château pour Jacob de la Gardie (1583-1652) ${ }^{10}$. De 1641 à 1647, en tant que conducteur, Tessin dressa des cartes de villes et repéra les cours d'eau dans le nord de la Suède; en 1646, il succéda à Simon de la Vallée au poste d'architecteur et byggmästere (architecte et directeur de chantier, littéralement "maitre de construction ») royal. Dès le milieu des années 1640 , nos deux architectes œuvraient donc pour l'élite suédoise, notamment en produisant des dessins d'architecture.

\section{Voyages d'études précurseurs}

5 Tessin comme de La Vallée furent les premiers architectes en Suède à bénéficier de voyages d'études sur le continent. Le 2 mai 1646, Jean de la Vallée se vit attribuer une bourse d'études pour un voyage de trois ans, et quitta Stockholm peu après avec l'ambassade de Magnus Gabriel de la Gardie (1622-1686). Deux lettres à Axel Oxenstierna nous informent sur son séjour parisien. Le 27 août 1646, il loge à Paris chez son grand-père et son oncle, les maîtres maçons Marin et Nicolas de la Vallée, et il étudie pour le compte de l'architecte Christophe Gamard. Le 25 janvier 1647, il poursuit ses études chez "plusieurs bons maîtres ", mais se plaint du coût de l'enseignement et des instruments nécessaires ${ }^{11}$. Chez son grand-père, il disposait d'une bibliothèque avec des livres classiques d'architecture, et par ses liens familiaux il a pu bénéficier d'accès privilégiés à des chantiers importants dans la capitale et ses environs. Nous ne savons rien sur ses activités à Rome, mais il y séjourna en avril 1649.

6 Quant à Nicodème Tessin, la mention de « Nic. Tessin de Stralsund en Poméranie ", sur une liste d'étudiants à l'université de Leiden du 14 septembre 1647, laisse supposer qu'il y effectua un premier séjour d'études ${ }^{12}$. Ceci ne correspond toutefois pas avec sa présence sur le chantier de la Maison de la Noblesse à Stockholm, où il supervisa les travaux sous les ordres de Heinrich Wilhelm du printemps 1647 jusqu'en $1648^{13}$. En mars 1651 un passeport, signé de la reine Christine, autorise Tessin à voyager en Allemagne, Italie, France et Hollande. À Rome, il fut apprenti auprès des architectes Girolamo et Carlo Rainaldi, participant ainsi à la conception de l'église Sant'Agnese in Agone. C'est peut-être à cette occasion que Tessin apprend à utiliser le dessin comme moyen d'expliquer et de déléguer l'exécution des idées de manière précise aux conducteurs de chantier, sans que la présence de l'architecte soit obligatoire ${ }^{14}$.

7 Parfaire ainsi leurs formations eut un retentissement immédiat sur les carrières de Tessin et de la Vallée. Premiers architectes en Suède à effectuer des voyages d'études sur le continent - et donc les seuls du métier dans ce pays à disposer de connaissances des modes européens de construction, non seulement théoriques mais aussi pratiques ils furent sollicités pour des projets avant même leurs retours. Dès lors, sans pouvoir être présents sur les chantiers, ils durent maitriser l'art de concevoir un projet en donnant des instructions dessinées et écrites, ne permettant pas de vérifier l'exécution des travaux. Des compétences alors également rares dans le nord de l'Europe. 


\section{Dessiner pour construire}

8 Jean de la Vallée fut rappelé à Stockholm au printemps 1650 pour préparer les célébrations du couronnement de la reine Christine (1626-1689), notamment avec la construction d'un arc de triomphe éphémère. Cette même année il fut promu architecte royal, une charge qui lui laissait moins de temps pour suivre les chantiers in situ. Il œuvrait aussi pour des particuliers, notamment en tant qu'architecte principal de Magnus Gabriel de la Gardie, qui était alors le plus grand constructeur et mécène d'art après la royauté suédoise. Très sollicité, de la Vallée se permit rapidement de refuser des commandes et même de se déplacer sur les chantiers. Certains projets exigeaient toutefois sa présence, tels la chapelle de Jacobsdal et le tombeau de Jacob de la Gardie dans l'église de Veckholm, où Jean de la Vallée fut payé pour « dessin et contrôle $»^{15}$.

Tessin abandonna aussi progressivement les chantiers. Dès son retour en Suède durant l'été 1653, il fut envoyé sur l'île d'öland pour superviser la modernisation de la fortification de Borgholm. Il fut ensuite transféré à Kalmar, où il participa à la préparation du déplacement de la ville et entama la construction de l'un de ses chefsd'œuvre : la cathédrale de la ville. Jusqu'au début des années 1660, il dut jongler entre ses activités pour la royauté en province et d'autres à Stockholm (pour la royauté et des particuliers), et se plaignit à plusieurs reprises du coût et des contraintes de ce mode de fonctionnement. Nommé architecte de la ville de Stockholm en 1661 et architecte du palais royal en 1663, Tessin n'œuvra alors plus que depuis Stockholm. Très sollicité par les mêmes particuliers que son confrère, comme lui, il refusait souvent de se déplacer sur les chantiers. Dans les années 1670, Tessin se permit même de réduire la fréquence de ses visites sur le chantier du château de la reine (Drottningholm), ne s'y rendant que deux fois par semaine ${ }^{16}$. De même, dans un envoi de dessins avec des explications écrites pour les aménagements au château d'Ekolsund, demandés par le propriétaire Claes Tott en 1666, Tessin explique que c'est à Tott de trouver un conducteur de travaux compétent. Trois ans plus tard, lorsque le commanditaire demanda des précisions sur la construction des cascades projetées dans les jardins, Tessin refusa de quitter Stockholm ${ }^{17}$ (Ill. 1). Un inventaire de 1666 spécifie qu'une pièce dans le grenier du palais Leuhusen à Stockholm, devait abriter une grande maquette du château d'Ekolsund. Le chercheur Claes Ellehag émet l'hypothèse que cet aménagement a servi de support aux discussions du projet de Tott avec son architecte ${ }^{18}$. 


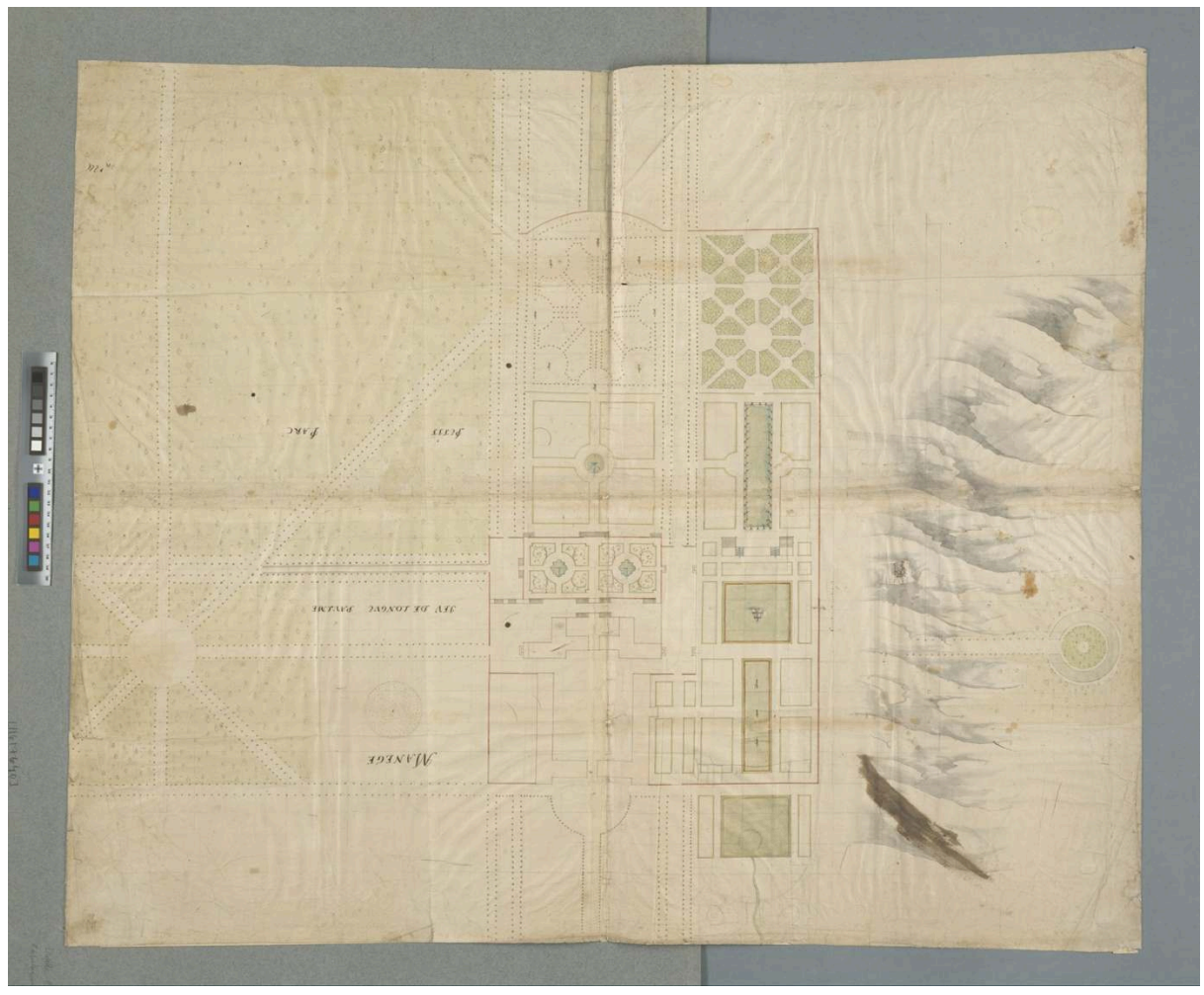

Avec indication d'échelle et dimensions aidant à l'installation de celles-ci sans la présence de l'architecte. Dessin attribué à Tessin l'Aîné par Börje Magnusson, collection d'Erik Dahlbergh, la bibliothèque Royale, Stockholm.

Bibliothèque Royale, Stockholm.

10 Le refus de suivre les chantiers in situ, correspondait à une évolution de l'idée défendue par Simon de la Vallée autour de 1640 -que l'architecte devait superviser les projets de construction de la conception jusqu'à l'achèvement. Procéder autrement permit de répondre à la forte demande de projets. Néanmoins, ne plus superviser l'exécution rendait plus libre l'interprétation des dessins par les équipes exécutantes, d'origines et compétences diverses, souvent des artisans locaux dirigés par des maîtres allemands, flamands, français, italiens ou baltes. Si l'essentiel de l'œuvre de Tessin et de la Vallée est imprégnée d'inspirations classiques plutôt sobres, l'exécution de détails de certains châteaux par ces artistes-artisans, tels Jacobsdal, Venngarn et Karlberg, rend les ensembles plus pittoresques et colorés que ce que montrent les dessins des deux architectes ${ }^{19}$.

11 Cette manière de fonctionner permit aussi aux architectes d'imposer leurs règles. Notamment en étant moins réactifs vis-à-vis de commanditaires difficiles. Ainsi lorsque Carl Gustaf Wrangel, un passionné d'architecture proposant constamment des changements, sollicita séparément nos deux architectes pour valider ses propres plans pour son palais à Stockholm en 1653, de la Vallée ne répondit qu'au bout de deux ans, suite à maintes relances et à l'envoi de cadeaux coûteux. Quant à Tessin, il ne présenta une maquette qu'en $1657^{20}$. Une autre raison de refus ou de manque de réactivité fut la difficulté, voire l'impossibilité d'être payés. Les deux architectes se plaignent de manière récurrente de leurs faibles revenus, alors qu'entre 1650 et 1680 leurs noms sont liés à une centaine de chantiers de toutes sortes. De la Vallée se porta partie civile dans deux procès pour non-paiement, lors desquels il se plaignit d'une double charge 
de travail en tant qu'architecte proprement dit mais aussi directeur de chantier, voire instructeur d'une main-d'œuvre non formée ${ }^{21}$. Parfois ce fut le fait des commanditaires (la royauté comme les particuliers), demandant des projets aux deux architectes pour une même construction, afin de comparer leurs propositions, voire de faire une synthèse des deux. Chacun intervenait d'ailleurs régulièrement sur des chantiers initiés par l'autre, notamment pour des projets trop ambitieux, freinés par les guerres ou par le manque de ressources. Ce qui explique les difficultés d'attribution et de datation de leurs projets, ainsi que les augmentations de coûts.

\section{Les ateliers de travail, les premières agences d'architecture en Suède?}

Il n'existe pas d'informations claires sur l'endroit où ces deux architectes concevaient leurs dessins et maquettes, mais nous savons qu'ils recevaient des clients chez eux. De par leurs fonctions auprès de la Royauté, il est plausible qu'ils aient disposé d'espaces dans le château royal ou dans un bâtiment annexe (tel était le cas au siècle suivant). Dès les années 1650 l'architecte du roi fut assisté par un copiste et un conducteur des travaux, et dix ans plus tard l'architecte du palais royal avait sous ses ordres un conducteur de chantier, un copiste et des assistants. Il disposait également d'un budget pour des fournitures, dont du papier, de l'encre et un cheval ${ }^{22}$. Dès lors une pièce du château fut dédiée à l'archivage des dessins d'architecture civile commandés par la couronne, qui constituèrent les débuts de la fameuse collection Tessin Hårleman (THC) aujourd'hui conservée au musée national à Stockholm ${ }^{23}$. Nous pouvons y imaginer l'aménagement de bureaux, au moins pour les copistes.

13 Tout laisse également penser que nos deux architectes disposaient d'espaces de travail à leurs domiciles. Le recensement de 1652 note « le maître constructeur [byggmestare] Johan Lavelée célibataire à Ladugårdslandet avec ses domestiques ${ }^{24}$ ». Ce qui signifie que de la Vallée logeait alors sur un grand terrain $\left(40000 \mathrm{~m}^{2}\right)$ dans la périphérie de Stockholm, obtenu par une donation de la reine cette même année. Dès 1653 et jusqu'aux années 1660 il y fit construire un bâtiment de pierre comprenant une taverne, des terrains de jeux (le premier parc d'attraction à Stockholm) et sans doute son logement (Ill.2). Il avait même l'intention d'y aménager une exposition d'habitations (boutställning). Selon l'historien de l'art Ellehag, il s'agirait de la plus originale de ses œuvres ${ }^{25}$. En 1653 il se maria avec la fille du maire d'Örebro, Maria Böös. Ils eurent dix enfants, et la famille semble avoir vécu ici jusqu'en 1682. Leur domicile était connu pour son accueil et son animation. Erik Dahlbergh (1625-1703), conducteur puis chef de la Fortification, un ami de Jean de la Vallée marié avec la nièce de son épouse, fut un habitué. Il semble crédible que Jean de la Vallée y eut un atelier : en 1654 il demandait à un agent de passer chez lui en fin de journée pour récupérer un dessin préparatoire ${ }^{26}$ (Ill. 3) ; et nous savons que dès cette époque, de la Vallée forma son frère Henri(k) de la Vallée (1634-1676), qui œuvrait comme ornemaniste avant d'être nommé ingénieur de la ville de Stockholm en 1667. Il est également possible qu'il ait initié son demi-frère Gustaf (1641-inc.) à l'art de la construction avant que celui-ci n'intègre l'administration de la Fortification. Dans les années 1660, les apprentis « ingénieurs » Johan Gerner (inc.-1663) et Mathias Spihler (1640-1690) furent nourris et logés dans la maison de leur maître. Spihler épousa l'une des filles de de la Vallée, et assista son beau-père en même temps qu'il œuvrait sous les ordres de Tessin et en tant 
qu'architecte indépendant ${ }^{27}$. Au moins deux des fils de la Vallée furent apprentis de leur père. Dès l'âge de 10 ans, Jonas (1657-1673), l'aîné, reçut une bourse d'état annuelle pour continuer son éducation dans l'art de la construction. Malheureusement il décéda en Italie lors de son voyage d'études. Son frère cadet, Christoffer Johan (1661-1700) conducteur à la Fortification, dit avoir reçu une instruction de son père en architectura civilis ex fundamento, en mathématiques et en fortification, avant d'étudier à l'université d'Uppsala. Ce fut probablement aussi le cas du fils cadet, Magnus Johan (1670-1728), qui devint officier dans cette même administration après des études à l'université d'Uppsala.

III. 2 : projet des jardins du château de Fredrikshov par le surintendant Carl Hårleman

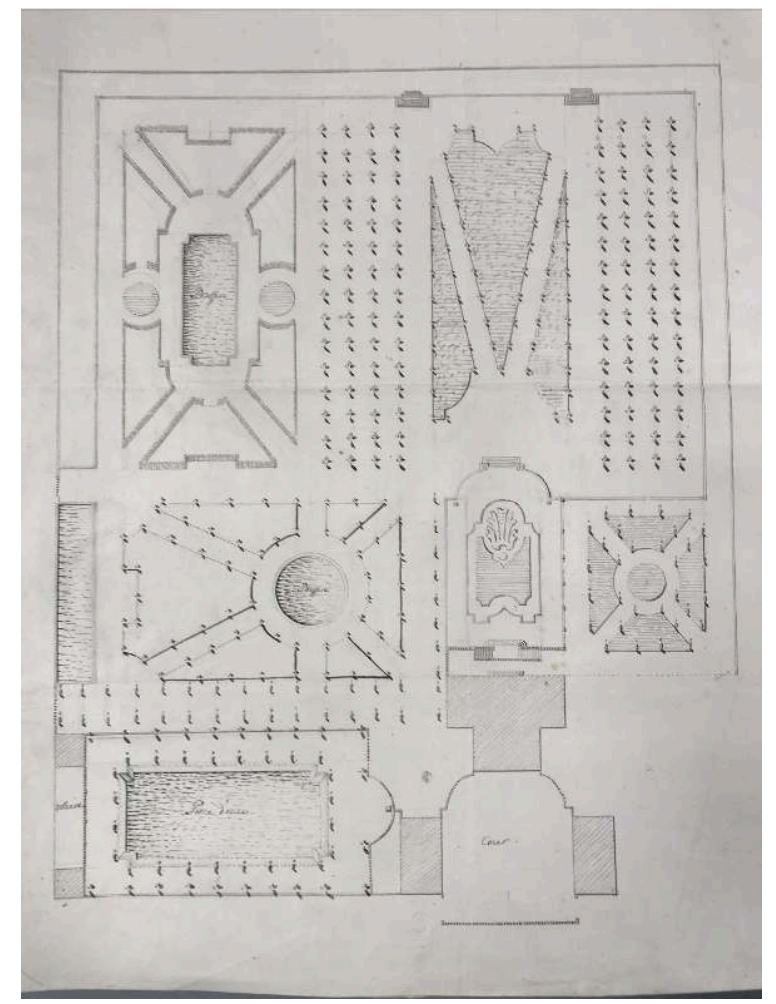

Un réaménagement de l'ancienne propriété de Jean de la Vallée dans les années 1730

Nationalmuseum THC 7637. Une partie des bâtiments existent encore aujourd'hui dans l'école du château de Fredrikshov.

Stockholm National Museum. 
III. 3 : plan d'ensemble du château d'Ekolsund avec les jardins, Jean de la Vallée

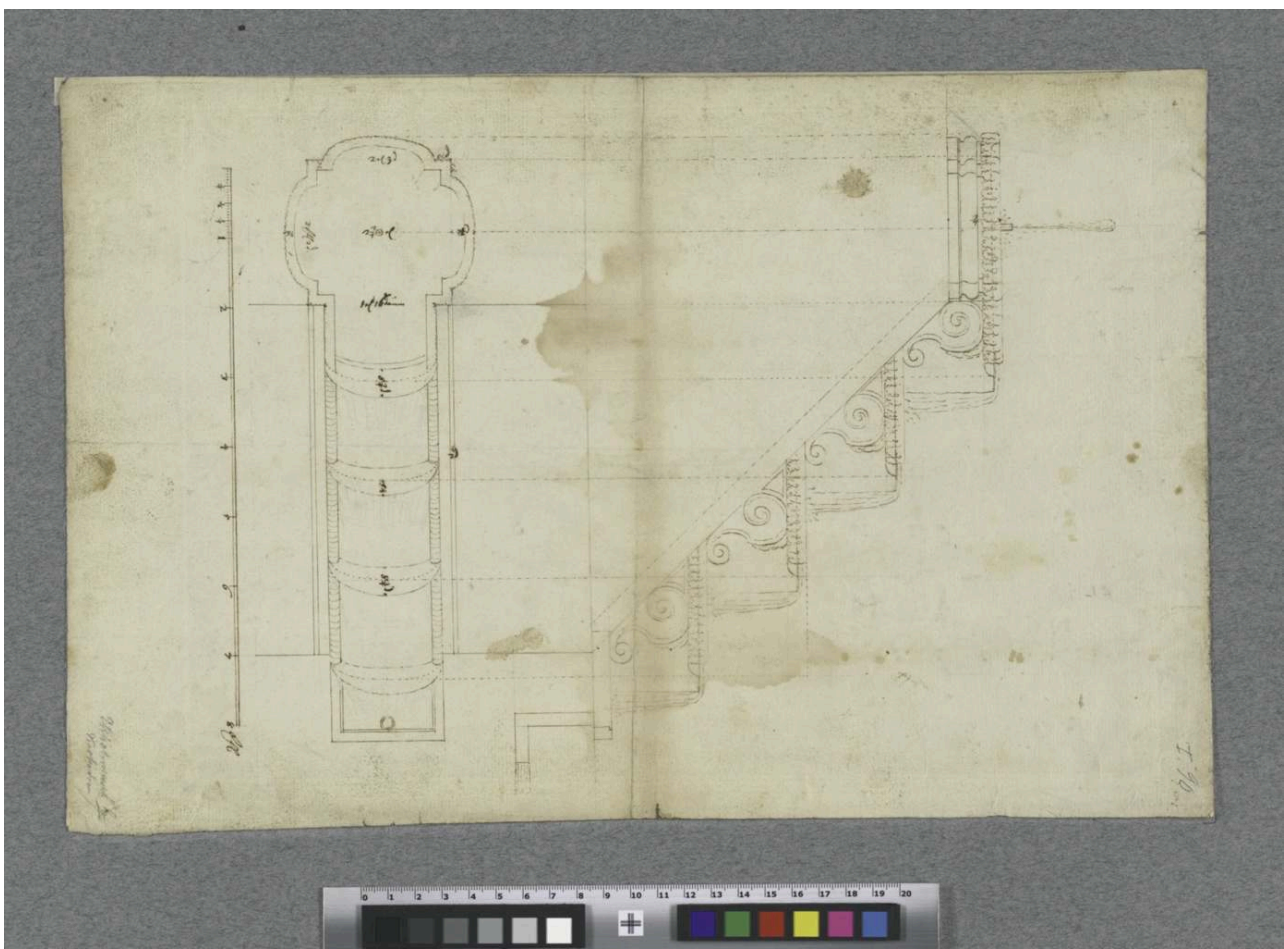

Dessin attribué à Jean de la Vallée par Börje Magnusson, collection d'Erik Dahlbergh, la bibliothèque Royale, Stockholm

Bibliothèque Royale, Stockholm.

En ce qui concerne Tessin, une lettre de 1663 stipule que Tessin reçut tant de visites prestigieuses pour des demandes de constructions privées dans son atelier, qu'il ne put tout réaliser ${ }^{28}$. Rien n'indique le lieu de cet atelier, mais comme pour son confrère il est plausible qu'il eut des bureaux à son domicile. Dès son retour en Suède, à l'été 1653 , Tessin se maria avec la veuve Maria Svahn, fille du maire de Västerås et mère de deux enfants. Ils eurent un fils, Nicodème Tessin le Jeune (1654-1728). La famille logeait au cœur du nouveau quartier de la vieille ville de Stockholm (Stora Nygatan 22) et en 1668 Tessin acheta une parcelle à l'adresse prestigieuse Skeppsbron 20, où il construisit face au port, entre 1670 à 1675 , un " palais ", qu'il dut rapidement vendre pour des raisons financières (Ill. 4). Nous connaissons une partie de son équipe: le conducteur de chantier Johan Peter Kristenius a pu recevoir une formation chez Tessin, certains dessins exécutés pour de la Gardie étant signés de lui ${ }^{29}$; nous savons aussi que l'architecte Johan Tobias Albinius (1635-79) œuvrait pour Tessin jusqu'en 1668 ${ }^{30}$, lorsqu'il fut remplacé par un Georg Tessin (peut-être un frère) et le beau-fils Abraham Wijnandtz (1644-1711) ${ }^{31}$; à partir de 1667, Matthias Spihler travaillait également sous les ordres de Tessin et le fils Nicodème assistait son père en tant que copiste dès ses 13 ans. Quand il étudia à l'université d'Uppsala, avant d'effectuer son premier voyage d'études en 1672, il fut remplacé par Magnus Gabriel Crell en tant que copiste (qui ensuite collabora avec Erik Dahlbergh). 


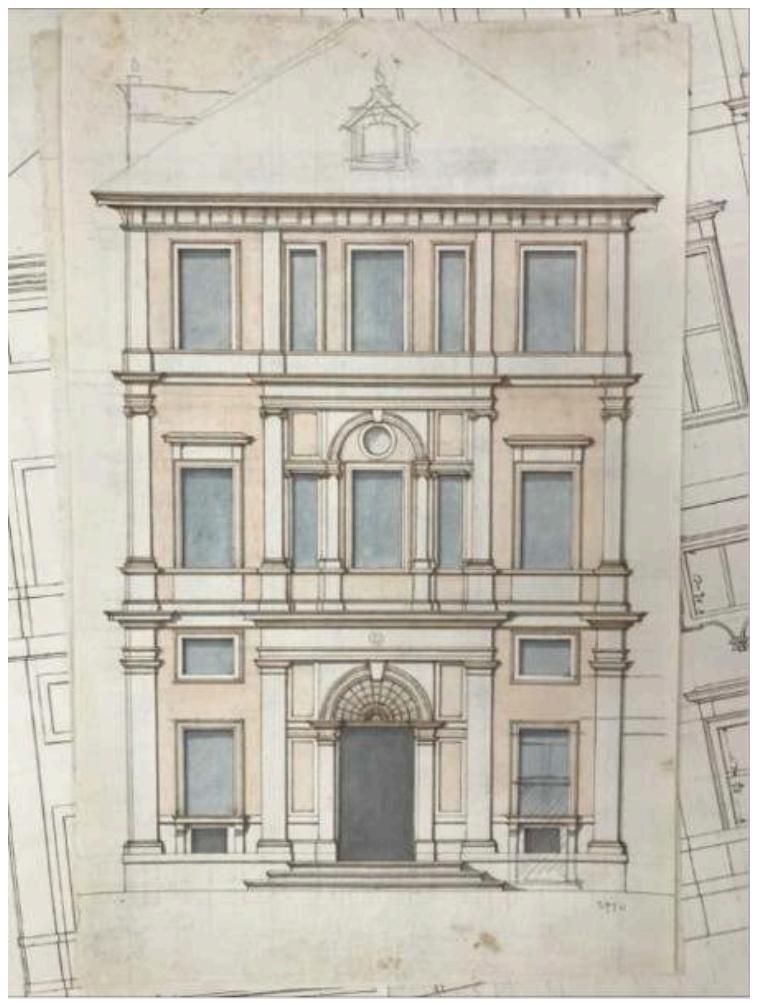

Par lui-même, construite à Skeppbron 20 dans les années 1670, Nationalmuseum, THC 2970.

Crédit : Stockholm National Museum.

15 Ainsi nos deux architectes furent assistés par des équipes, en partie constituées de leurs familles. Les fonctions de chacun et l'organisation de la préparation des dessins sont impossibles à cerner, mais il apparaît que de la Vallée prit plus de temps à produire que son confrère. Tessin déléguait peut-être mieux ou cela est tout simplement dû au cumul de métiers de Jean de la Vallée. Les descriptions et les dessins conservés témoignent de personnalités antinomiques. Tessin est décrit par ses contemporains comme un homme calme, sincère, diligent, serviable et plutôt aimable. Selon lui l'architecte devait "inventer ce qui sert à un édifice utile et orné et mettre les mains des autres au travail, ce qui est le bon et le meilleur enseignement pour les hommes de l'artisanat ${ }^{32}$. "Souvent réalisés à l'aide d'un compas et d'une règle, les dessins de sa main témoignent d'un esprit rationnel. Les représentations des projets sont précises, l'échelle est souvent indiquée sur les plans, les élévations et les coupes (Ill. 5), un procédé alors rare en Suède. Quant à Jean de la Vallée, lors d'un procès en 1685, il se définit en tant « qu'artiste libre ${ }^{33}$. » Les dessins de sa main, plus rares, sont plus artistiques mais moins précis que ceux de Tessin. Il s'agit surtout de plans d'ensemble mais aussi de quelques élévations et coupes (Ill.6). L'esprit précurseur et mercantiliste de Jean de la Vallée, de même que sa franchise, semblent avoir heurté certains de ses contemporains, et ses bonnes relations avec l'élite ont pu provoquer des jalousies. La place centrale des livres dans l'apprentissage et la pratique de l'architecture est confirmée par des bibliothèques, relativement bien fournies aux domiciles de nos deux architectes. Il est d'ailleurs probable qu'ils eurent aussi accès à celles de la royauté, de leurs collègues et clients principaux, dont la plupart furent des grands bibliophiles. 
III. 5 : coupe du château de Sjöö

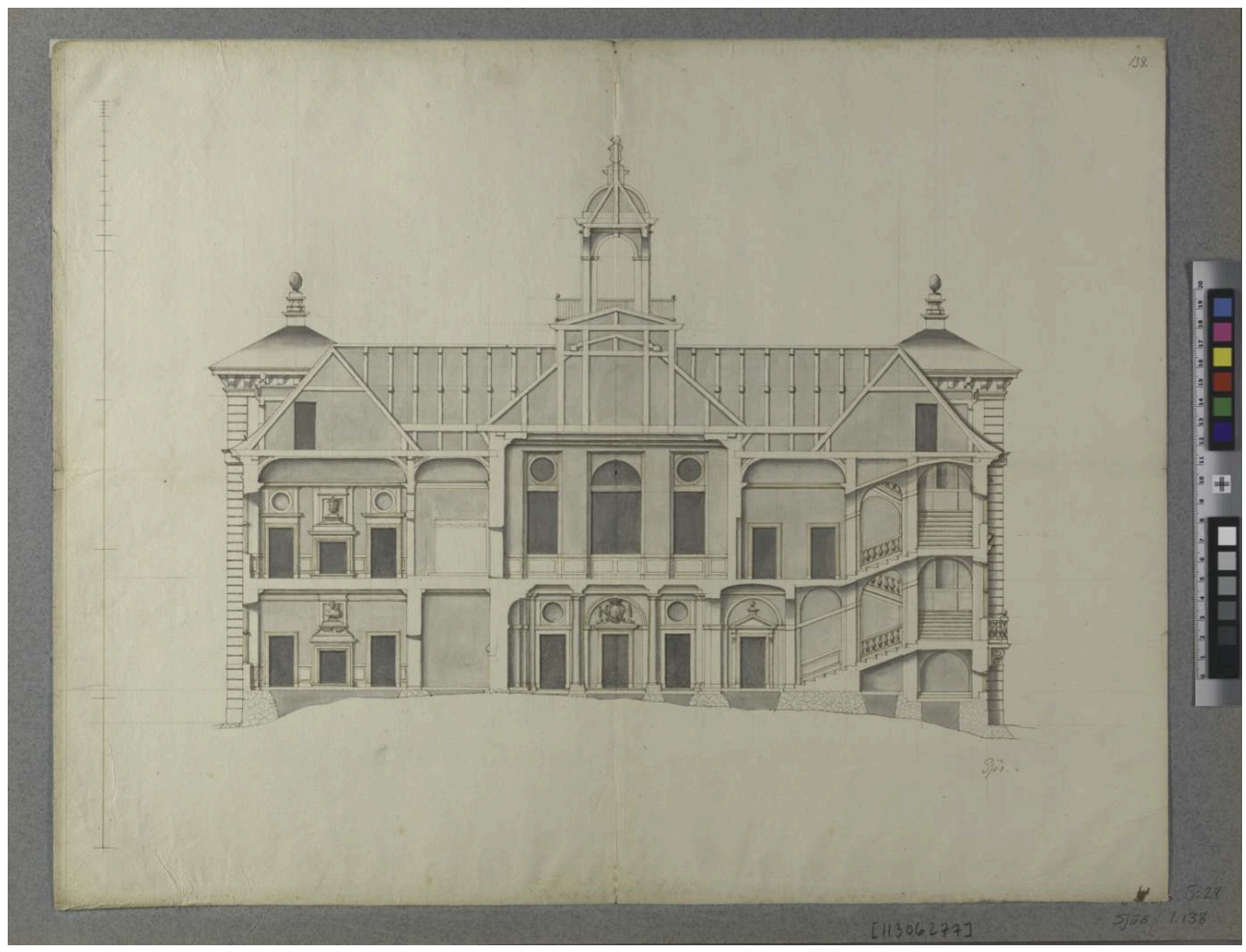

Dessin attribué à Tessin l'Aîné par Börje Magnusson, collection d'Erik Dahlbergh, la bibliothèque Royale, Stockholm.

Bibliothèque Royale, Stockholm. 


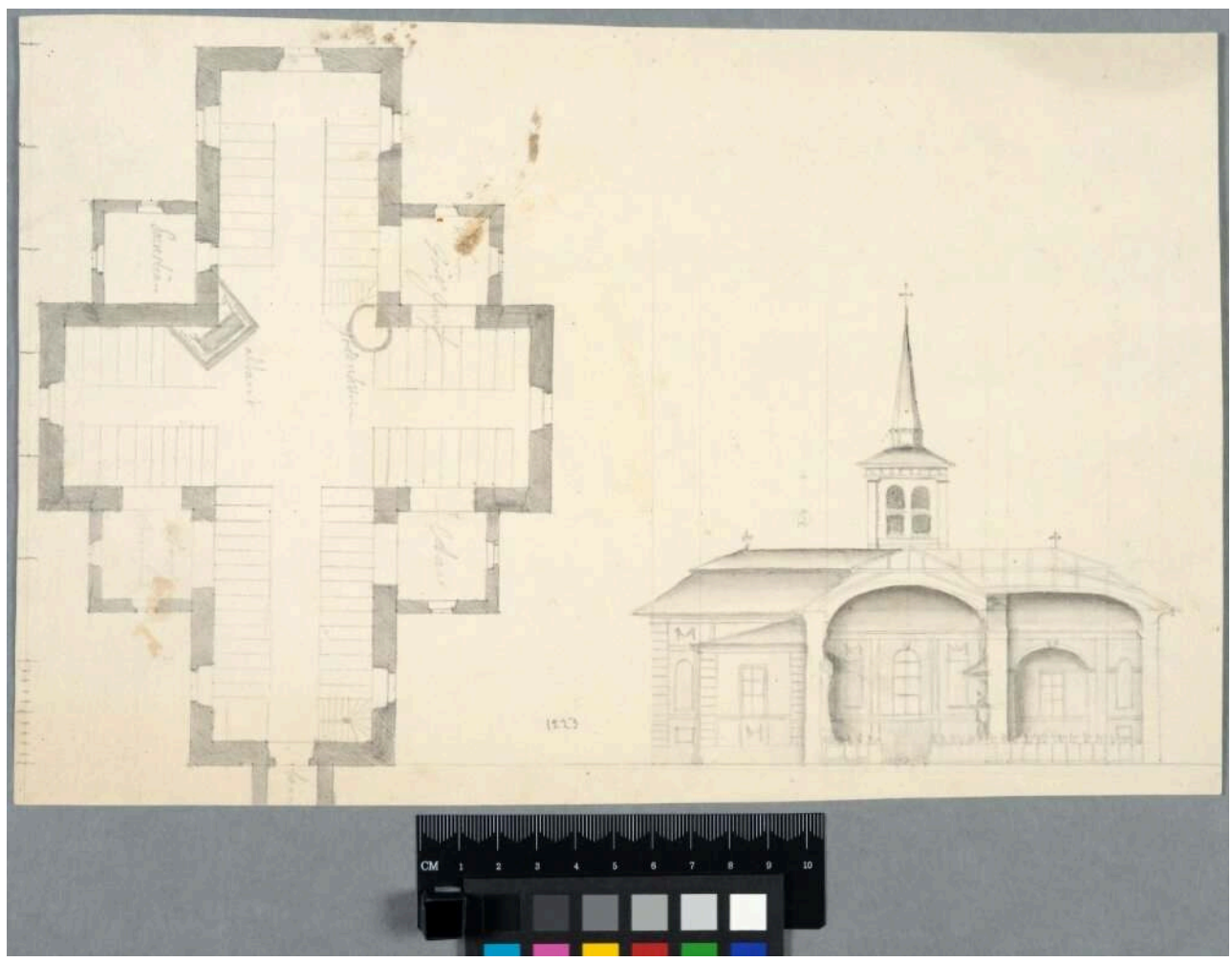

Plan et coupe, Jean de la Vallée 1653, NMH, THC 1223.

Photographie : Cecilia Heisser

\section{De l'architecteur à l'architecte}

Le fait que pendant vingt ans Tessin ait œuvré sous les ordres de son cadet Jean de la Vallée, architecte principal et directeur des bâtiments, contribua certainement à des rivalités, voire des jalousies. Le second prônait l'instauration d'un surintendant (luimême) qui devait notamment superviser l'architecte de la ville (Tessin) et approuver tous les dessins d'autres architectes et maîtres maçons. Cette fonction ne devint une réalité qu'après sa mort, avec la nomination de Tessin le Jeune en 1697. Néanmoins la famille Tessin prit le dessus dès 1671, quand Jean de la Vallée fut élu maire de la commission des bâtiments - Byggnadskommissionen. Car le cumul de cette fonction, avec celui de commisarius des manufactures depuis 1662 pour la chambre de commerce, Kammarkollegium - avec la responsabilité de surveiller l'artisanat et les industries du pays - ralentit son activité intense d'architecte ${ }^{34}$.

17 L'obtention du titre de comte en 1674 confirma la promotion sociale de Tessin, une distinction que reçut de la Vallée dix-huit ans plus tard. Ses armoiries arborent le lion (la force et la solidité), le lys (la beauté) et le coquillage (la commodité) ${ }^{35}$. Ce qui peut être interprété comme un rappel vitruvien du prestige de sa profession. Quant au sceau de Jean de la Vallée, utilisé dès janvier 1643, outre ses initiales il présente trois étoiles (l'apprenti, le compagnon, le maître?), un compas (l'outil du grand créateur, l'architecte), enlacé par des serpents (la sagesse) dans une couronne de laurier (la gloire). Il ressemble à celui de son père, composé de même d'un compas avec un 
serpent mais dans un bouclier (force, justice, prudence, protection?), renouant ainsi avec leurs origines de maîtres maçons ${ }^{36}$.

Avec leurs collaborateurs, protecteurs et clients, les architectes Tessin et de la Vallée contribuèrent donc de manière significative à la promotion du statut de la profession et de la pratique de l'architecture dans leur patrie adoptive. La Grande réduction instaurée par le roi Charles XI dès 1679, durant laquelle le pouvoir de la noblesse fut considérablement réduit et où une grande partie des domaines (auparavant distribuées par la royauté) fut confisquée, provoqua l'arrêt ou la simplification de maints chantiers. Le richissime Magnus Gabriel de la Gardie notamment fut complètement ruiné, ce qui fut également le destin de nombreuses autres familles dans son entourage. Malgré cela, après le décès de Nicodème Tessin l'Aîné en 1681, la famille, tel les Mansart en France, vit son œuvre, des projets commencés et des desseins, comme un héritage commun à transmettre. Son fils et son beau-fils reprirent des dessins et des chantiers inachevés. Le legs de Jean de la Vallée est moins évident à cerner. Leurs manières de pratiquer le métier d'architecte furent prolongées par la création d'une Surintendance des bâtiments du roi dès 1697. Au milieu du XVIII siècle, ce corps prit la forme d'une agence nationale centralisée supervisant toutes les conceptions architecturales en Suède - publiques, privées et religieuses. Malheureusement nous ne disposons pas de sources appropriées concernant l'organisation pratique du travail de ces équipes, qui sont à l'origine d'une production très conséquente, souvent attribuée comme étant l'œuvre des seuls Surintendants. ${ }^{37}$

\section{NOTES}

1. Quelques travaux fondateurs sur ce sujet : August Hahr, Konst och konstnärer vid Magnus Gabriel De la Gardies hof, Uppsala, Akad. Bokhandel, 1905, 200 p. Osvald Sirén, Gamla Stockholmshus af Nicodemus Tessin d. ä. och några samtida byggnader, Stockholm, Nordstedt \& söner, 1912-13, 2 vol. Ragnar Josephson, Stadsbyggnadskonst $i$ Stockholm intill 1800, Uppsala, Almqvist, 1918. Ragnar Josephson, Nicodemus Tessin d.y.: tiden, mannen, verket, Stockholm, 1930-1931, 2 vol. Sans compter de nombreuses autres publications de ces derniers, ainsi que de Sten Karling, Tord 0:son Nordberg, et d'autres chercheurs auxquels cet article fera référence, ne pouvant donner ici une bibliographie exhaustive.

2. Kristoffer Neville, Nicodemus Tessin the Elder: architecture in Sweden in the Age of Greatness, Turnhout, Brepols, 2009, 275 p., et Claes Ellehag, Jean de la Vallée kunglig arkitekt, Lund, Signum, 2003, $251 \mathrm{p}$.

3. Paru en 1715 de manière inachevée, l'ouvrage est posthume à Dahlbergh. Börje Magnusson, «Sweden illustrated : Erik Dahlbergh's "Suecia antiqua et hodierna" as a manifestation of imperial ambition", Allan Ellenius (dir) Baroque dreams: art and vision in Sweden in the era of greatness, Stockholm, Almqvist \& Wiksell, 2003, p. 32-59.

4. Neville, op cit., p. 25.

5. Josephson, Tessin d.y. ... , op. cit., p. 15. 
6. Barbro Flodin, "Jean de la Vallées första verk. Hans utbildning och verksamhet före utlandsresan 1646 - sedd mot bakgrunden av samtida svenska förhållanden", Konsthistorisk tidskrift, 1977, n 1-2, p. 123-143.

7. Linnéa Rollenhagen Tilly, « De maître maçon à architecte, Simon et Jean de la Vallée », Olga Medvedkova (dir), Les Européens: ces architectes qui ont bâti l'Europe (1450-1950), Bruxelles, Peter Lang, 2015, p. 107-127.

8. Flodin, op. cit., p. 125. Cet article comprend une bibliographie plus exhaustive au sujet de la famille de la Vallée.

9. «Jean Lavalet måtte och medh något benficio aff Cronan blifwa försedd, om så mycket bättre haffwa tillfelle at Continuera hantwerket, och giöra sigh till Cronans tienst i framtiden capabel ", le 24 janvier 1643, cité par Flodin, op. cit., p. 128.

10. Flodin, op. cit., p. 126.

11. Ibid., p. 139.

12. Johan Eriksson, Peter Liljenstolpe, Sjöslott: Nicodemus Tessin dä. och Johan Gabriel Stenbock som aktörer vid ett stormaktstida slottsbygge, p. 24.

13. Neville, op. cit., p. 43.

14. Ibid., p. 117.

15. Hahr, op. cit., p. 26, Ingrid Rosell, Ulriksdals slottskapell, Stockholm 1976, p. 16.

16. Néville, op. cit., p. 157.

17. Ibid., p. 158.

18. Claes Ellehag, Palatsen i Stockholm under stormaktstiden, Lund, 1998, p. 75.

19. Ingrid Rosell, Venngarns slottskapell, Stockholm, Almqvist \& Wiksell, 1988, p. 35.

20. Ellehag, op. cit.1998, p. 102-111.

21. Werner Pursche, Timmermansämbetet i Stockholm före 1700, bihang, Stockholm, Stellan Ståls tryckeri, 1979, p. 65-72.

22. Ellehag, op. cit., 2003, p. 30.

23. Neville, op.cit., p.157. Martin Olin, «The drawings of the Tessin collection in the Nationalmuseum, Stockholm : the decorative arts ", Les marques de collections, dir. Peter Fuhring. Dijon 2010, p. 87-95.

24. Ellehag, op. cit. 2003, p. 204, «byggmestare Johan Lavalée, ogift tillsammans med sitt tjänstefolk på Ladugårdslandet”. En 1653 il obtint un privilège pour préparation de vin et de distillation.

25. Ellehag, op. cit. 2003, p. 204-206.

26. Ellehag, op. cit. 2003, p. 30.

27. Claes Ellehag, Kronan och adelns arkitekt. Matthias Spihler ca 1640-1690, Lund, Signum, 2010, 223

p.

28. Lettre d'un collaborateur de C.G. Wrangel, Ellehag op. cit. 2003, p. 31.

29. Ingrid Rosell, Veckholms kyrka, Stockholm, Almqvist \& Wiksell, 1974, p. 36.

30. Claes Ellehag, Johan Tobias Albinus (ca 1635-1679): arkitekt $i$ Sverige och Baltikum: en arkitekturhistorisk och biografisk skildring, Stockholm, 2011, $101 \mathrm{p}$.

31. Neville, op. cit., p. 151.

32. Josephson, op. cit., p. 30 ; Ellehag, op. cit. 2003, p. 28.

33. Ellehag, op. cit. 2003, p. 29.

34. Hahr, op. cit., p. 93.

35. Göran Lindahl, "En arkitektkarriär i maktens följe", Tessin. Nicodemus Tessin d.y. Kunglig arkitekt och visionär, Stockholm, Nationalmuseum, 2002, p. 12.

36. Flodin, op. cit., p. 129 ; Ellehag op. cit. 2003, p. XXXIII.

37. Cf. par exemple Bo Vahlne, Frihetstidens inredningar på Stockholms slott. Om bekvämlighetens och skönhetens nivåer, Stockholm, Balkong 2012, 597 p. et Linnéa Rollenhagen Tilly, Carl Johan Cronstedt, arkitekt och organisatör, Stockholm, Balkong, 2017, 254 p. 


\section{RÉSUMÉS}

Cet article présente l'organisation du travail des deux architectes phares en Suède entre 1650 et 1680 : Nicodème Tessin l'Aîné (1615-1681) et Jean de la Vallée (1624-1696) : par leur notoriété, ils contribuèrent à un nouveau statut et à une nouvelle gestion de leur profession, et il s'agira de montrer en quoi cela nous permet d'arguer qu'ils furent les premiers architectes avec des agences en Suède.

This paper presents how the two "star" architects in Sweden from 1650 to 1680, Nicodemus Tessin the elder (Stralsund 1615 - Stockholm 1681) and Jean de la Vallée (Paris 1624 - Stockholm 1696) organised their work. How thanks to their notoriety they contributed to a new status and management of their profession. And how this makes it possible to argue that they were the first architects with firms in Sweden.

Der Beitrag beschäftigt sich mit der Arbeitsorganisation der beiden bedeutendsten in Schweden zwischen 1650 und 1680 tätigen Architekten: Nicodemus Tessin der Ältere (1615-1681) und Jean de la Vallée (1624-1696). Dabei soll der Frage nachgegangen werden, wie die beiden Akteure ihre jeweilige Bekanntheit dazu nutzten, ihrem Berufsstand zu einem neuen Status und einem neuen Umgang zu verhelfen, und inwiefern dies zu der Aussage berechtigt, dass es sich um die ersten so zu bezeichnenden "Architekten" mit echten "Architekturbüros" in Schweden handelt.

\section{INDEX}

Mots-clés : Suède, architecte, pratique, dessin, chantier, agence, atelier, instruction

\section{AUTEUR}

\section{LINNÉA ROLLENHAGEN-TILLY}

Docteur en histoire de l'art, Linnéa Rollenhagen Tilly est spécialiste de l'histoire de l'architecture et de la construction à l'époque moderne. Ses recherches portent sur les transferts de savoir-faire et de modèles entre la France et la Suède. Auteure d'un livre sur le surintendant des bâtiments en Suède Carl Johan Cronstedt (1709-1777), son activité, sa bibliothèque et sa collection - Carl Johan Cronstedt, arkitekt och organisatör, Stockholm, Balkong, 2017, elle a également publié une vingtaine d'articles. Outre son enseignement en histoire et cultures architecturales en tant que Maître de conférences associé à l'ENSA Paris la Villette, elle dispense de cours dans plusieurs programmes universitaires américains à Paris. 